\title{
Activation of blood coagulation in chronic urticaria: pathophysiological and clinical implications by Dr. Massimo Cugno et al.
}

\author{
Marco Cicardi
}

Received: 23 February 2010/Accepted: 1 March 2010/Published online: 27 March 2010

(C) SIMI 2010

Cugno et al. [1] reviewing the topic of chronic urticaria (CU) highlight the role of blood coagulation in the pathophysiology of this disease. This is an innovative view that represents the major breakthrough of the last few years to unravel the poorly understood mechanisms underlying the pathogenesis of urticaria lesions.

Previous important discovers in this field are depicted in a figure of the paper that reports the chronology of the key events of CU pathophysiology investigation in the last two decades. The first important discover dates back to the 1980s, when Grattan et al. described the possible autoimmune origin of the disease in about half of $\mathrm{CU}$ patients in whom the intradermal injection of autologous serum caused a wheal and flare reaction. Subsequently, the presence of histamine-releasing autoantibodies directed against $\operatorname{IgE}$ or the alpha-subunit of the high-affinity $\operatorname{IgE}$ receptor (FceRI) was found in about $30-35 \%$ of CU patients. The skin reaction to autologous serum was ascribed to the presence of functionally active autoantibodies to FceRI and to other less-characterized histamine-releasing factors as activation of the complement system by $\operatorname{IgG}$ autoantibodies against the alpha-subunit of the FceRI that has been shown to enhance histamine release. The autoimmune background, in a consistent proportion of CU patients, is further supported by the frequent association with autoimmune thyroiditis [2] and by anecdotal reports of the association between $\mathrm{CU}$ and other autoimmune disorders, such as Graves disease, type 1 diabetes and celiac disease.

Although the presence of histamine-releasing autoantibodies accounts for CU pathomechanism in almost half of

M. Cicardi $(\bowtie)$

Dipartimento di Scienze Cliniche "Luigi Sacco",

Università di Milano, Ospedale L. Sacco Milano, Milan, Italy

e-mail: marco.cicardi@unimi.it the patients, the pathomechanisms involved in the other half of patients remain not determined. The studies on blood coagulation can partially fill this gap. Hence, it is known that activation of the coagulation cascade leads to generation of vasoactive substances such as thrombin which stimulate endothelium to increase vascular permeability [3, 4]. CU patients show an activation of coagulation cascade through the tissue factor expressed by eosinophils infiltrating CU lesional skin. Thus, it is possible to hypothesize that the eosinophil is a leading actor in the CU scenario.

The activation of coagulation has been described also in other diseases characterized by inflammation, including other cutaneous disorders, such as bullous pemphigoid and angioedema due to $\mathrm{C} 1$ inhibitor deficiency [5-8], and a wide spectrum of systemic inflammatory diseases, such as rheumatoid arthritis [9] inflammatory bowel diseases [10] and sepsis [11]. This should not necessarily lead to consider activation of the coagulation as a nonspecific phenomenon, but rather to think that different diseases share, at different sites, a common intermediate step in their pathophysiology. Thus, in the skin microenvironment of CU and bullous pemphigoid, eosinophils express TF which activate coagulation and generate vascular permeability mediators. In angioedema due to C1-inhibitor deficiency, another skin disease characterized by increase in vascular permeability, there is an activation of blood coagulation [7, 8], but edema is mediated by bradykinin generated upon activation of the contact system. An obvious concern with these views comes from the observation that markers of thrombin generation like $\mathrm{F} 1+2$ are increased in several prothrombotic conditions, such as disseminated intravascular coagulation [12], deep venous thrombosis [13] and endotoxinemia [14] that are not characterized by urticaria or edema. Therefore, we can conclude that thrombin 
activation per se is not enough to generate these symptoms, but it probably acts synergistically with other vasoactive substances released by concomitant activation of complement, contact system or mast cells. In fact, the vasoactive effect of bradykinin and histamine can be potentiated by the effect of thrombin, as the three substances represent distinct classes of endothelial cell agonists that increase vascular permeability by different mechanisms [3, 4].

It is apparently contradictory that patients with $\mathrm{CU}$ or deficiency of C1-INH are not reported to be at risk for thrombotic events, despite an increased activation of the coagulation cascade. To reconcile this apparent contradiction, we may reconsider the coagulation system in the same way as it was done for the complement system. Analogous to coagulation, the complement is a cascade system made of zymogens converted into active enzymes by proteolysis. Described as the effector of antibody-mediated cell lysis, the function of complement has been revisited. Today, it is part of the innate immune system due to the proinflammatory functions pertaining to cell lysis and even more to the biological activities of the active fragments released by the proteolytic activation of its components. Evidence for a role of coagulation in inflammatory diseases without apparent thrombotic effect suggests that the activation of coagulation components may be pathophysiologically relevant independent of thrombus formation. Nevertheless, a thrombotic risk is part of other inflammatory diseases. In bullous pemphigoid, which is characterized by a strong involvement of eosinophil and tissue factor-mediated activation of coagulation, an increased thrombotic risk has been observed; therefore, these aspects should be considered in future studies [5].

A final consideration, looking at coagulation studies in $\mathrm{CU}$, is the potential use of anticoagulant treatment in this disease. Indeed, there are reports indicating that heparin and vitamin $\mathrm{K}$ antagonists may be beneficial in $\mathrm{CU}$ patients resistant to conventional therapy with antihistamines. These scattered reports can not lead to conclusion, but highlight the need for controlled clinical trials aimed to evaluate the efficacy of anticoagulants in selected $\mathrm{CU}$ patients who do not respond to antihistamines.

\section{References}

1. Cugno M, Marzano AV, Asero R, Tedeschi A (2009) Activation of blood coagulation in chronic urticaria: pathophysiological and clinical implications. Intern Emerg Med. doi:10.1007/ s11739-009-0333-5

2. Leznoff A, Sussman GL (1989) Syndrome of idiopathic CU and angioedema with thyroid autoimmunity: a study of 90 patients. J Allergy Clin Immunol 84:66-71

3. Schaeffer RC Jr, Gong F, Bitrick MS Jr, Smith TL (1993) Thrombin and bradykinin initiate discrete endothelial solute permeability mechanisms. Am J Physiol 264:H1798-H1809

4. Ehringer WD, Edwards MJ, Miller FN (1996) Mechanisms of alpha-thrombin, histamine, and bradykinin induced endothelial permeability. J Cell Physiol 167:562-569

5. Marzano AV, Tedeschi A, Fanoni D, Bonanni E, Venegoni L, Berti E, Cugno M (2009) Activation of blood coagulation in bullous pemphigoid: role of eosinophils, and local and systemic implications. Br J Dermatol 160:266-272

6. Marzano AV, Tedeschi A, Spinelli D, Fanoni D, Crosti C, Cugno M (2009) Coagulation activation in autoimmune bullous diseases. Clin Exp Immunol 158:31-36

7. So AK, Varisco PA, Kemkes-Matthes B et al (2003) Arthritis is linked to local and systemic activation of coagulation and fibrinolysis pathways. J Thromb Haemost 1:2510-2515

8. Kume K, Yamasaki M, Tashiro M et al (2007) Activations of coagulation and fibrinolysis secondary to bowel inflammation in patients with ulcerative colitis. Intern Med 46:1323-1329

9. Liaw PC, Esmon CT, Kahnamoui K et al (2004) Patients with severe sepsis vary markedly in their ability to generate activated protein C. Blood 104:3958-3964

10. Cugno M, Cicardi M, Bottasso B, Coppola R, Paonessa R, Mannucci PM, Agostoni A (1997) Activation of the coagulation cascade in C1-inhibitor deficiencies. Blood 89:3213-3218

11. Cugno M, Zanichelli A, Bellatorre AG, Griffini S, Cicardi M (2009) Plasma biomarkers of acute attacks in patients with angioedema due to C1-inhibitor deficiency. Allergy 64:254-257

12. Teitel JM, Bauer KA, Lau HK, Rosenberg RD (1982) Studies of the prothrombin activation pathway utilizing radioimmunoassays for the F2/F1 + 2 fragment and thrombin-antithrombin complex. Blood 59:1086-1097

13. Boneu B, Bes G, Pelzer H, Sie P, Boccalon H (1991) D-Dimers, thrombin antithrombin III complexes and prothrombin fragments $1+2$ : diagnostic value in clinically suspected deep vein thrombosis. Thromb Haemost 65:28-31

14. van Deventer SJ, Büller HR, ten Cate JW, Aarden LA, Hack CE, Sturk A (1990) Experimental endotoxemia in humans: analysis of cytokine release and coagulation, fibrinolytic, and complement pathways. Blood 76:2520-2526

\section{Conflict of interest None.}

